\title{
Nanoscale
}

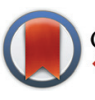

CrossMark

Cite this: Nanoscale, 2015, 7, 20435

\section{Structure vs. excitonic transitions in self- assembled porphyrin nanotubes and their effect on light absorption and scattering $\dagger$}

\author{
Oriol Arteaga, ${ }^{\star a}$ Adolf Canillas, ${ }^{a}$ Zoubir El-Hachemi, ${ }^{\text {b }}$ Joaquim Crusats ${ }^{\mathrm{b}}$ and \\ Josep M. Ribób
}

\begin{abstract}
The optical properties of diprotonated meso-tetrakis(4-sulphonatophenyl)porphyrin (TPPS 4 ) J-aggregates of elongated thin particles (nanotubes in solution and ribbons when deposited on solid interfaces) are studied by different polarimetric techniques. The selective light extinction in these structures, which depends on the alignment of the nanoparticle with respect to the polarization of light, is contributed by excitonic absorption bands and by resonance light scattering. The optical response as a function of the polarization of light is complex because, although the quasi-one-dimensional structure confines the local fields along the nanotube axis, there are two orthogonal excitonic bands, of $\mathrm{H}$ - and $\mathrm{J}$-character, that can work in favor of or against the field confinement. Results suggest that resonance light scattering is the dominant effect in solid state preparations, i.e. in collective groups (bundles) of ribbons but in diluted solutions, i.e. with isolated nanotubes, the absorption at the excitonic transitions remains dominant and linear dichroism spectra can be a direct probe of the exciton orientations. Therefore, by analyzing scattering and absorption data we can determine the alignment of the excitonic bands within the nanoparticle, i.e. of the orientation of the basic 2D porphyrin architecture in the nanoparticle. This is a necessary first step for understanding the directions of energy transport, charge polarization and non-linear optical properties in these materials.
\end{abstract}

Received 19th August 2015 Accepted 27th October 2015 DOI: 10.1039/c5nr05633k www.rsc.org/nanoscale porphyrin (TPPS ${ }_{4}$ ). Although the porphyrin building blocks are achiral, the self-assembled J-aggregates show an intrinsically chiral structure that leads to chiral electronic transitions, i.e. to circular dichroism at the corresponding electronic transitions. The electronic and optical properties of the porphyrin are drastically modified as a result of its self-assembly into a nanoparticle, because dipole-dipole interactions among the constituent molecules cause an energy shift of the absorption bands compared to the monomer and the transfer of the local excitation to form a Frenkel exciton, i.e. the charge separation in the electronic transition through many dipole-dipole interacting porphyrins. Furthermore, the homogeneous ordering of these oscillators within a nanoparticle yields an oscillator coupling between a large number of excitons. ${ }^{4,5}$

There are two types of excitonic coupling between the porphyrin monomer units: the so-called J-bands, a consequence of the side-to-side coupling of transition dipoles, showing a red shift of the Soret- and Q-bands of the porphyrin $(489 \mathrm{~nm}$ and $707 \mathrm{~nm}$, respectively, compared to $433 \mathrm{~nm}$ and $644 \mathrm{~nm}$ of the monomer) and also a blue-shifted Soret band $(\sim 420 \mathrm{~nm})$, called $\mathrm{H}$-band, that is characteristic of porphyrin face-to-face coupling ( $\pi$-stacking). Currently, there is increasing interest 


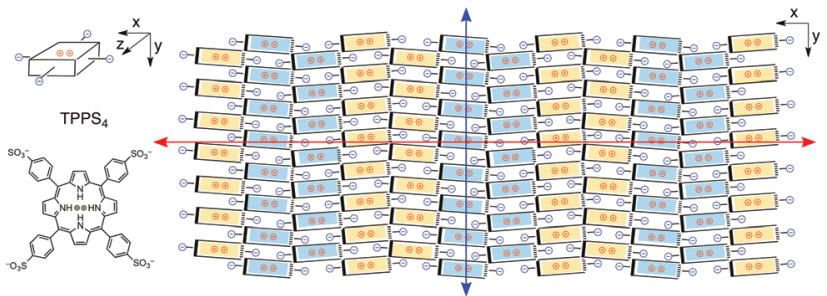

Fig. 1 Porphyrin architecture on a plane. The excitons are shown by the double headed arrows ( $\mathrm{H}$-exciton, blue; J-exciton, red).

in the study of the role of $\mathrm{H}$ - and J-excitonic interactions in a soft-matter light harvesting system ${ }^{6,7}$ and for potential optoelectronic applications. ${ }^{8}$

Recently, the structure of a chiral porphyrin mosaic sheet of $\mathrm{TPPS}_{4}$ J-aggregates has been revealed by diffraction techniques. ${ }^{9}$ This structure, shown in Fig. 1 , takes into account the orthogonality between $\mathrm{J}$ - and $\mathrm{H}$-excitonic bands, which was experimentally inferred in previous reports. ${ }^{10,11}$ The nanoparticles of the $\mathrm{TPPS}_{4}$ J-aggregates show different morphologies that can be formed by mono-, bi- or multilayered structures. The most interesting ones assemble in long and thin structures that in the solution are hollow, single-walled nanotubes ${ }^{12-14}$ and when deposited on dry substrates collapse into bilayered ribbons. We use an experimental procedure adapted from ref. 14 that allows the preparation of solutions containing only nanotubes and, therefore, it is possible to assess that the optical properties of these samples correspond to a specific group of mesoscopic forms, i.e. nanotubes in the solution and ribbons by solution casting on a solid/air interface.

The porphyrin monomers self-assemble into the $2 \mathrm{D}$ sheet of Fig. 1. This sheet is flexible enough to roll into a nanotube when the aggregation reaches an adequate length, in a similar way that a graphene sheet would wrap into a single walled carbon nanotube.t The orientation of the J- and H-excitons with respect to the nanotube axis depends, actually, on the rolling direction. The aim of this paper is to verify experimentally the nature and orientation of these transitions in the nanoparticles by means of polarimetric methods that study the absorption and scattering contributions to the nanotube extinction properties. We find that the overall optical response arises from the reduced dimensionality of the particles combined with the excitonic bands that are embedded in their structure. These results also allow for a better understanding of the chiroptical phenomena arising when stirring a nanotube solution.

$\$$ Recent diffraction results ${ }^{9,14}$ show that the porphyrin rings are coplanar with the monolayer. The closing of the sheet structure of Fig. 1 into a nanotube must imply some structural relaxation. However, the structural modification in the transition from a planar nanoparticle to a nanotube is probably not very significant because it is not detected by changes in the UV/Vis absorption spectra (using routine spectrometric techniques).

\section{Experimental}

\subsection{Polarimetric measurements}

Polarimetric measurements were made with two different instruments: a spectroscopic Mueller matrix polarimeter based on four photoelastic modulators ${ }^{15}$ and a Mueller matrix microscope based on the continuous dual rotating compensator technique $^{16}$ (further experimental details are available in the ESI $\dagger$ ). The first instrument was used to analyze solutions containing $\mathrm{TPPS}_{4}$ nanotubes, in both transmission and scattering configurations using a $10 \mathrm{~mm}$ path length cuvette. The microscope was used to study solid-state preparations of the same substances in which it was possible to observe ribbon bundles. Narrow bandpass filters at $500 \mathrm{~nm}$ and $420 \mathrm{~nm}$ were used in the microscope to study, respectively, the J-excitonic and $\mathrm{H}$-excitonic transitions. The Mueller matrix data were analyzed using the analytic inversion $\operatorname{method}^{17}$ to calculate, among others, the linear extinction (LE) of the samples. Here we will report LE as the difference between the horizontal and vertical extinction in the instrument's coordinate system; moreover the component at $45^{\circ}$ is also measured, so it is possible to recover the absolute direction of extinction. We use the terminology linear extinction, instead of the more common term linear dichroism (LD), because LD refers only to the differential absorption of light for two orthogonal polarizations, while we assume that LE is more general and can include both the scattering and absorption contributions.

\subsection{Sample preparation}

Solutions of J-aggregate nanoparticles were prepared by injecting $250 \mu \mathrm{l}$ of a mother solution of the diprotonated porphyrin hydrochloride form TPPS $_{4} \cdot 2 \mathrm{HCl}(2 \mathrm{mM}-8 \mathrm{mM}$ of the zwitterionic porphyrin according to ref. 18) into $1 \mathrm{ml}$ of $0.01 \mathrm{M} \mathrm{HCl}$. The procedure leading to solutions composed quasi-exclusively of straight particles (ribbon/nanotubes) was adapted from ref. 14 and is based on the slow injection of the mother solution using a capillary needle (Hamilton syringe, $0.7 \mathrm{~mm}$ external diameter needle, injection time $5 \mathrm{~s}$ ) into the inner portion of the acid water solution. The mother solutions were prepared from the hydrochloride porphyrin equilibrated for 24 hours or from fresh eluted concentrated solutions of the zwitterionic porphyrin prepared by column ion exchange (protonated form) of the porphyrin tetrasodium salt free of salts. More common preparation methods in which the mother solution is added to the acid solution under gentle stirring lead to solutions containing a relatively high proportion of straight nanoparticles but also a significant amount of round small particles and plate-like particles.

Films for Mueller matrix microscopy were prepared from concentrated solutions showing only long straight particles $(>1 \mu \mathrm{m})$. Solution drops were deposited on an inclined glass slide in order to obtain some alignment between the nanoparticle bundles. They were left to dry at normal room temperature and humidity condition.

Diluted solutions for optical measurements in $1 \mathrm{~cm}$ path length cuvettes were obtained by the dilution of $7 \mu \mathrm{l}$ of the 


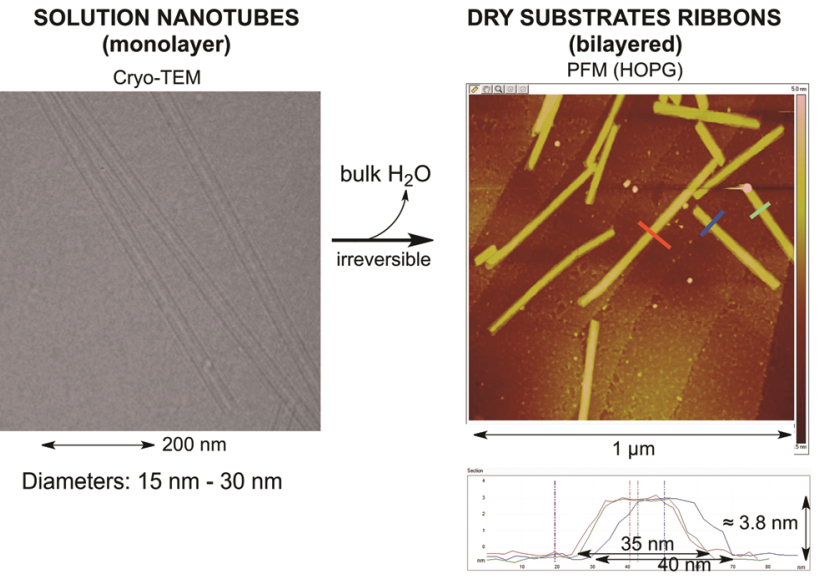

Fig. 2 Transition from hollow single wall nanotubes seen from the cryo-electron microscopy image to ribbons when deposited on dry substrates (solid/air interface) as observed in the PFM image.

above described nanotube solutions in $1250 \mu \mathrm{l} 0.01 \mathrm{M} \mathrm{HCl}$ and $0.4 \mathrm{M} \mathrm{NaCl}$ water solution. The dilution in acidic solution $(\mathrm{pH} 2)$ in the absence of $\mathrm{NaCl}$ would lead to slow deaggregation of the porphyrin aggregates. The stability of the nanotubes in this dilution procedure was controlled by Peak Force Microscopy (PFM). PFM methods are described in the ESI. $\dagger$

\subsection{Nanotubes $v s$. nanoribbons}

When the solution with nanotubes is deposited on a dry solid substrate and the surrounding bulk water is eliminated, the nanotubes collapse to bilayered ribbons as is shown in Fig. 2. This morphology variation leads to a change from the elastic behavior of the isolated nanotubes in a diluted solution to a plastic behavior of the bilayered ribbons. Details of this will be published elsewhere. As the bilayered ribbons originate from the collapse of a single nanotube, their two superposed layers must show the same architecture and orientation.

\section{Results}

Rayleigh scattering is normally not considered for nanoscale objects because it is expected to be extremely weak. ${ }^{19}$ However there is a resonant enhancement when the photon energy matches or is near an electronic transition and this effect is very strong when there is an intense coupling among the chromophore units, ${ }^{4,19}$ as is characteristic for the TPPS $_{4}$ family of J-aggregates. Resonance light scattering (RLS) is well known to be strong for aggregates with a large volume, ${ }^{4,19}$ and it can be further enhanced when thin nanoparticles assemble into bundles. Bundling occurs due to side-by-side interactions between the highly polarized porphyrin walls, leading to bundles of aligned nanotubes in which the LE effects increase with the thickness of the bundle, as is shown in Fig. 3. The linear extinction at $500 \mathrm{~nm}$ is very strong and it is clearly oriented along the axis of the ribbon bundles (Fig. 3). This result is in agreement with near field polarized microscopy measurements recently reported in ref. 20. At $420 \mathrm{~nm}$ the linear extinction becomes much weaker (is reduced by a factor of $\sim 10$, as shown in Fig. 3c), but it has the opposite sign with respect to the result at $500 \mathrm{~nm}$ because, in this case, it is oriented perpendicular to the ribbon axis.

The results in Fig. 3 showing that, for the J-aggregate transition, maximum extinction occurs for light parallel to the axes of the ribbon bundles could be indicative that the J-transitions are parallel to the nanotube axis. However this picture is not necessarily correct, because we have to consider that, in the observed bundles, RLS and not absorption is the predominant contribution to the extinction. The cross section of scattering $^{19}$ is given by:

$$
\sigma_{\text {sca }}=\frac{k^{3}}{6 \pi}|\alpha|^{2}=\frac{k^{3}}{6 \pi}\left(\alpha_{\mathrm{r}}^{2}+\alpha_{\mathrm{i}}^{2}\right)
$$

where $k$ is the wavevector of light, $k=2 \pi / \lambda$, and $\alpha_{\mathrm{r}}$ and $\alpha_{\mathrm{i}}$ are, respectively, the real and imaginary parts of the polarizability of the aggregates. Note that for nanotubes the scattering cross section scales as the third power of the wavevector, while for spherical particles it scales as the fourth power. ${ }^{21}$ The absorption cross section is

$$
\sigma_{\mathrm{abs}}=k \alpha_{\mathrm{i}}
$$

and so, unlike the scattering cross-section, it only depends on the imaginary part of the polarizability.

LE arises due to anisotropy in the polarizability of the porphyrin nanoparticles. This anisotropy can be contributed by two different factors: by the relative orientations of the J-excitons and H-excitons in the porphyrin sheet, and also by the quasi one-dimensional nature of the particle, which introduces a strong shape-induced anisotropy. For example, the strong scattering efficiency for light polarized along the nanotube axis is a well-known characteristic of carbon nanotubes; $;^{22-24}$ while, on the other hand, light polarized perpendicular to the nanotube axis loses efficiency because its polarizability is significantly reduced due to screening. This local-field or "antenna effect" strongly reduces the response whenever the electric-field vector of the optical radiation lies perpendicular to the nanotube. Interestingly, if J-excitons are perpendicular (or near perpendicular if chiral closing occurs), they act as very efficient charge transporters, screening this perpendicular polarized radiation by allowing charges to build up at each side of the nanotubes as is schematically represented in Fig. 4a. Likewise, H-excitons only allow charge transfer in the axial direction, and the charge builds up only at the ends of the nanotube and the screening is much less intense (Fig. 4b). This excitonic arrangement occurs if the porphyrin sheet in Fig. 1 rolls along the $x$ direction.

To understand better the contributions of absorption and scattering to the extinction, we performed spectroscopic transmission and scattering measurements in a diluted solution (where the nanotubes can be considered to be isolated one from another). Scattered light could be detected in a rather 

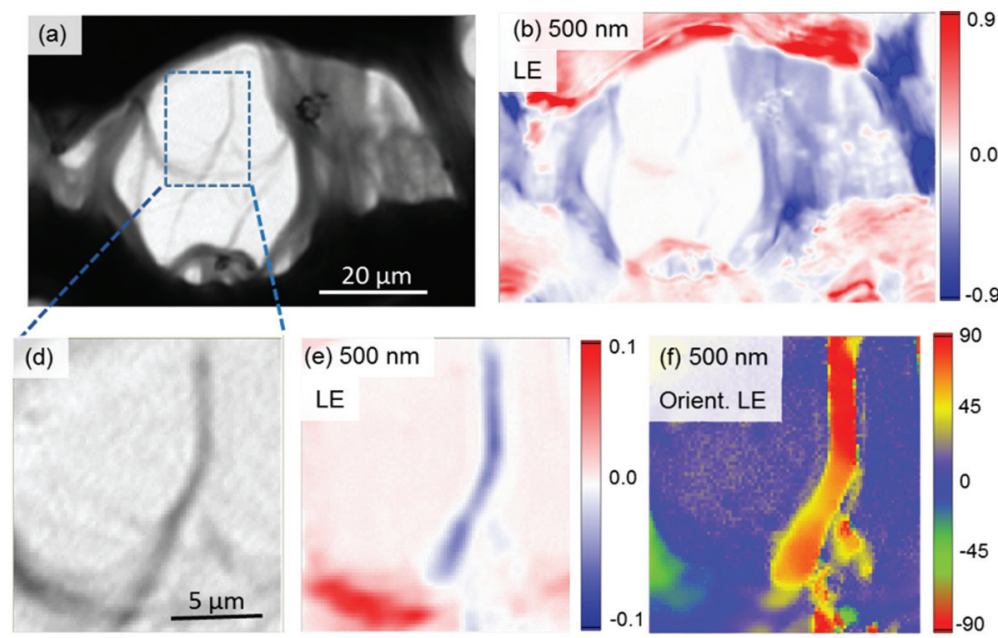

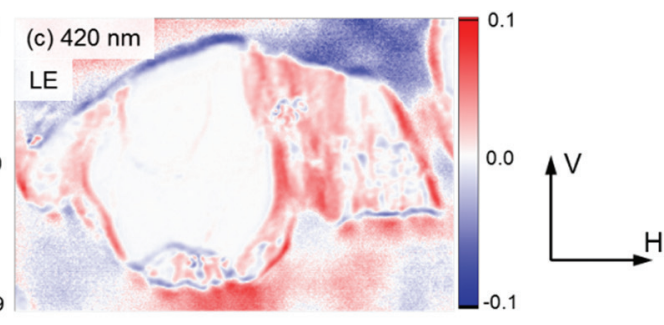

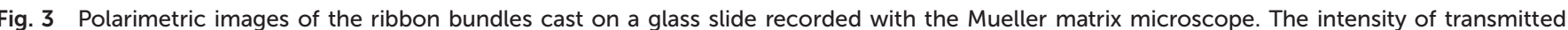

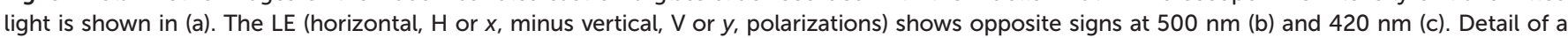


(in degrees) of the maximum LE. At $500 \mathrm{~nm}$ the extinction takes place along the bundle axes while at $420 \mathrm{~nm}$ it is orthogonal.
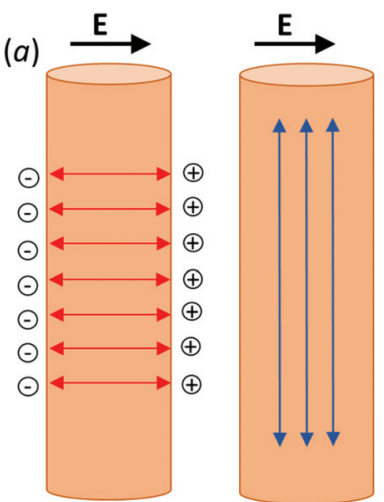

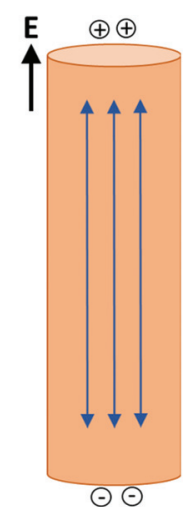

Fig. 4 Enhanced field screening due to the J-excitons (red arrows) for an electric field perpendicular to the nanotube axis (a). When the electric field is parallel to the nanotube axis (b), the $\mathrm{H}$-excitons (blue arrows) can only accumulate positive charges (holes) or negative charges (electrons) at the ends of a nanotube and the J-excitons do not contribute to the charge transfer. An analogous scheme holds for bilayered ribbons formed from nanotube collapsing.

broad range from approximately $410 \mathrm{~nm}$ to $540 \mathrm{~nm}$ (Fig. 5, top). The extinction bands measured in a transmission experiment for the same solution (Fig. 5, bottom) show two well distinctive orthogonal J-aggregate and $\mathrm{H}$-aggregate peaks, but they are clearly narrower than for the solid state sample. This suggests that transmission experiments in solution are mainly probing the absorption contribution (narrow peaks) because, if the light lost by scattering was the dominating contribution to the total extinction, broadened peaks should be expected also in transmission. Absorption peaks are narrow because they depend only on the imaginary part of the polarizability, but the scattering losses also depend on the real part of the polarizability that behaves anomalously in the vicinity of an absorption band, leading to broadened spectral peaks and to a small energy shift. This is the reason why the negative J-excitonic band is broadened in the solid state and slightly blueshifted, while the positive H-band is red-shifted.

It is also interesting to observe that there appears to be an anomalous behaviour in the scattering measurement (Fig. 5, top) between $475 \mathrm{~nm}$ and $515 \mathrm{~nm}$. This is the interval of wavelengths where the J-aggregate absorption appears to be dominant $(\mathrm{LE} \simeq \mathrm{LD})$ in the diluted solutions. The $90^{\circ}$ scattering configuration favors the scattering of vertically polarized light at all wavelengths (since scattering always produces linearly polarized light perpendicular to the scattering plane) but, in this interval range, the scattering experiment is also affected by the fact that the solution is absorbing more vertical polarization than horizontal polarization, in agreement with the sign of LE measured in transmission. As will be discussed later, this is related with the orientation taken by the nanotubes inside the cuvette due to unavoidable vertical convention flows induced by small temperature differences.

\section{Discussion}

Polarimetry measurements suggest that the optical response of the solid preparations is governed by the scattering contribution while, in diluted solutions, absorption is dominant. The reason for the different behaviour between the solid samples and solutions has to be related with the presence of bundles and is perhaps favored by the bilayered character of the ribbons compared to the monolayered nanotubes. Bundling perturbs the electronic structure of the nanotubes or ribbons due to side-by-side interaction between the highly polarized porphyrin sidewalls. Studies in single walled carbon 


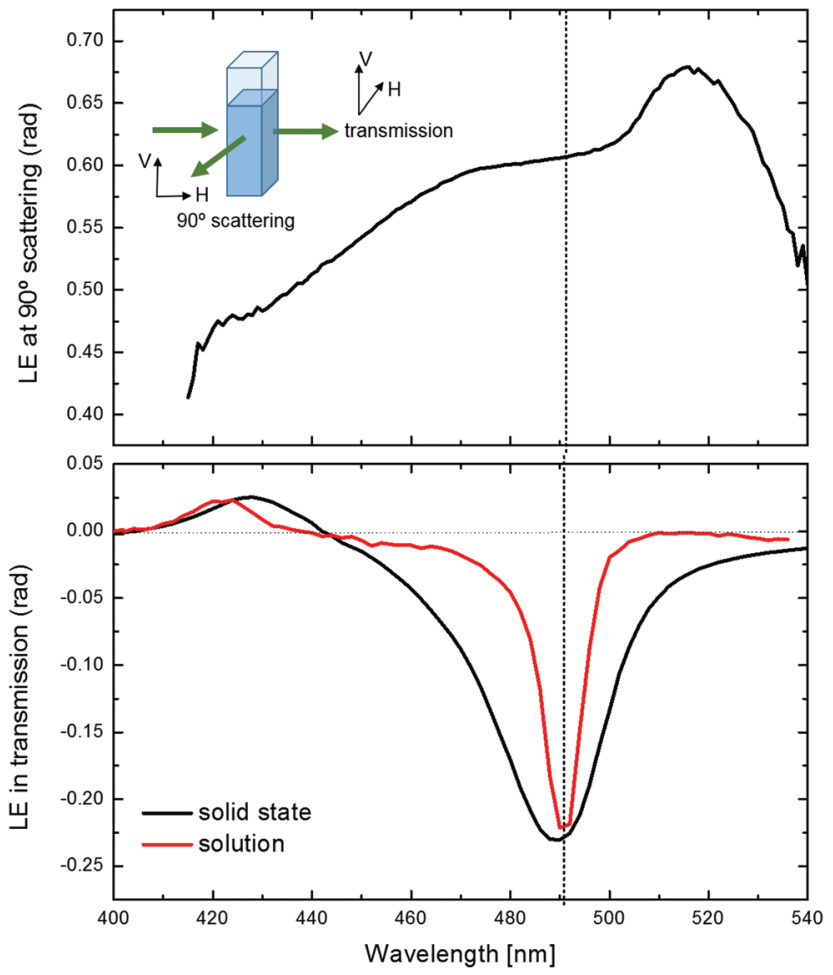

Fig. 5 Top: LE for the $90^{\circ}$ scattering of a solution of porphyrin aggregates (nanotubes). Bottom: LE for the same solution in transmission and compared with the LE of a solid sample (data corresponding to the solution have been scaled by a factor of $\sim 10$ to reach the same magnitude as in the solid state). The anomalous decrease of the scattering LE around $490 \mathrm{~nm}$ coincides with the J-aggregate band, which is only sharp for the transmission measurement in the solution.

nanotubes ${ }^{25}$ have shown that bundling produces an enhanced screening of the intratube Coulomb interaction (Fig. 4) and an intertube exciton tunneling. All this leads to a stronger antenna effect. No such effects are possible in the studied solutions because they contain isolated nanotubes with diameters between $15 \mathrm{~nm}$ and $30 \mathrm{~nm}$. The polarizability $(\alpha)$ of the elongated aggregates scales linearly with their volume and therefore, according to eqn (1), the amount of scattering will depend on the square of the volume of the aggregate, while the absorption will scale linearly. Therefore, the RLS efficiency will strongly depend on the particle size. We conclude that the larger concentration of extended aggregates in the solid state preparations, which is further enhanced by the strong bundling between particles, leads to stronger RLS effects than in diluted solutions.

In previous reports we have shown that, for this type of supramolecular aggregate, there is a circular dichroic sign induction by the effect of hydrodynamic torques. ${ }^{11,26,27}$ The main characteristic of those experiments is that switching the swirling direction inverts the circular dichroism (CD) spectra (Fig. 6c). To explain this phenomenon, it is necessary to relate the sign of the hydrodynamic torque in the region of measurement with the orientation of the nanotube in the
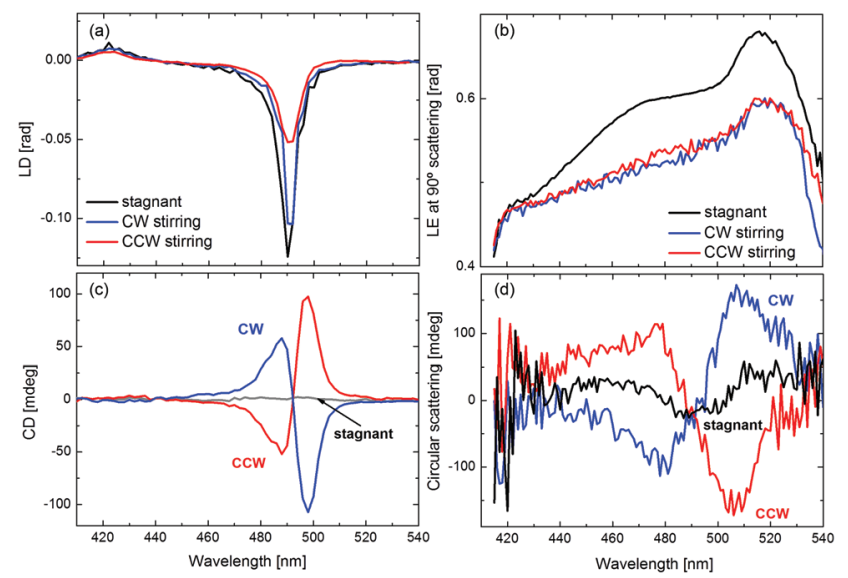

Fig. 6 Comparison of the linear and circular anisotropies of a nanotube solution under stirring and stagnant conditions. (a) Shows that there is an increase of LD in the transmission measurement when stirring, while (b) shows an analogous comparison but in terms of the LE observed for $90^{\circ}$ scattering. (c) Shows the induction of opposite patterns of CD for clockwise (CW) and counter clockwise (CCW) stirring. (d) Shows the differential circular scattering for $\mathrm{CW}$ and $\mathrm{CCW}$ stirring measured at the $90^{\circ}$ scattering configuration.

flow as well as with the ordering of the excitonic bands in the nanotube. Here we discuss only the orientation of the excitons in the nanotube as originated from the closing of the basic sheet of the J-aggregate structure (Fig. 1). A complete discussion on the origin of the transient $\mathrm{CD}$ signal originated from the stirring vortex derived will be published elsewhere. Under the stirring vortex, the magnitude of LE increases with respect to stagnant conditions (Fig. 6a) and the J-exciton band takes negative LE values. This result, together with the analysis of the velocity gradients occurring at the central region of the cuvette (where the spectra were recorded), allowed us to infer that nanotubes had to be predominantly standing horizontally (i.e. orthogonal to the cuvette axis) in the vortex flow. In the diluted nanotube solutions, even when absorption is the main contribution to the extinction, resonant scattering measurements still offer an alternative way to check for the orientation. To assess this, we repeated the $90^{\circ}$ measurement scheme of Fig. 5 but under clockwise (CW) and counter-clockwise (CCW) stirring conditions. The results are given in Fig. 6b. Here, contrary to what happened in transmission, the magnitude of the linear extinction decreased when stirring. The theory of light scattering from a thin cylinder states that scattering is more efficient when the incoming polarization is parallel to the cylinder axis, i.e. a cylinder standing horizontally in the cuvette should scatter more horizontal polarization than vertical polarization. Hence, less vertically polarized light reaches the detector, leading to the negative (i.e. decreasing) contribution to the linear extinction that we observe when stirring. These observations confirm that the nanotubes stand horizontally under gentle vortex stirring. 
The differential circular extinction at $90^{\circ}$ scattering for CW and CCW stirring is shown in Fig. 6d. The signs of the characteristic bisignated bands are opposite to those observed for transmission (Fig. 6c). This result is not too surprising, since it means that, if for example, with CW stirring, left-CPL has the highest extinction in transmission, scattered light will show a predominant lack (i.e. extinction) of right-CPL since this is the handedness that is more transmitted through the sample. In this case we can also observe that the scattering bands are broader than their transmission counterparts. This is another indication that absorption, and not differential light scattering, is the main contribution to the CD signals observed in transmission.

Our measurements confirm that the predominant orientation of the nanotubes is horizontal in the stirring vortex with the H-excitonic band probably oriented axially and the J-excitonic band radially. This is in agreement with the primordial sheet rolling into a nanotube through the $\mathrm{J}$ direction, as we had anticipated from the microscopy measurements. This rolling direction probably yields a more stable structure because it is the principal direction where the electrostatic, hydrogen bonds and hydrophobic effects act to form the J-aggregate. $^{3}$ The axial direction originates from weaker $\pi$-stacking forces but, as is common in self-assembly and in the crystallization of flat organic compounds, it corresponds to the direction of faster growth. In fact, nanotube ripening and growth at the expense of monomeric and small J-aggregate particles transform, after some weeks, short nanotubes (100 nm$300 \mathrm{~nm}$ ) into several $\mu \mathrm{m}$ long nanotubes of the same diameter.

\section{Conclusion}

We have been able to correlate experimentally the structure of the nanotubular J-aggregates with the orientations of the excitonic transitions. It is of special significance that the J-aggregate band at $489 \mathrm{~nm}$ contributes to the charge transfer necessary for the screening of perpendicular radiation, generating a strong structure-induced anisotropy in the polarizability. This is a second level of anisotropy that is generated by the rolling of the porphyrin sheet into a quasi-one dimensional nanotube and that is enhanced by the assembly in bundles. However, the porphyrin sheet already contains directions of anisotropy given by the mutually orthogonal $\mathrm{H}$ - and J-excitonic transitions. Series of polarimetric transmission and scattering measurements have allowed us to discern among absorption and scattering contributions to the extinction and, therefore, parse the two levels of anisotropy in these self-assembled nanostructures.

The porphyrin nanotubes act as excitonic antennas or photon concentrators for visible light with the particularity that they have both, axial and radial, transitions allowed in the structure. This is a unique characteristic when compared to other one-dimensional materials, such as inorganic nanowires or carbon nanotubes, and represents a clear advantage to funnel the photon excitations towards specific locations, especially when they assemble in large bundles. Future studies should focus on the preparation of oriented collective nanoparticle microstructures (for example, by alignment with microflow techniques) and on interfacing such antenna structures with optoelectronic devices to explore uses in photovoltaics and photodetectors.

\section{Acknowledgements}

This work was funded by the project CTQ2013-47401-C2-1 (MINECO) and forms part of the COST Action CM1304. O. A. acknowledges funding from the European Commission (Marie Curie IIF Fellowship PIIF-GA-2012-330513).

\section{References}

1 T. Kobayashi, J-aggregates, World Scientific, Singapore, vol. 2, 2012.

2 F. Würthner, T. E. Kaiser and C. R. Saha-Möller, Angew. Chem., Int. Ed., 2011, 50, 3376-3410.

3 B. J. Walker, Ph.D. thesis, Massachusetts Institute of Technology, 2011.

4 P. J. Collings, E. J. Gibbs, T. E. Starr, O. Vafek, C. Yee, L. A. Pomerance and R. F. Pasternack, J. Phys. Chem. B, 1999, 103, 8474-8481.

5 N. Micali, F. Mallamace, A. Romeo, R. Purrello and L. Monsù Scolaro, J. Phys. Chem. B, 2000, 104, 5897-5904.

6 D. M. Eisele, D. H. Arias, X. Fu, E. A. Bloemsma, C. P. Steiner, R. A. Jensen, P. Rebentrost, H. Eisele, A. Tokmakoff, S. Lloyd, K. A. Nelson, D. Nicastro, J. Knoester and M. G. Bawendi, Proc. Natl. Acad. Sci. U. S. A., 2014, 111, E3367-E3375.

7 Y. Wan, A. Stradomska, S. Fong, Z. Guo, R. D. Schaller, G. P. Wiederrecht, J. Knoester and L. Huang, J. Phys. Chem. C, 2014, 118, 24854-24865.

8 F. C. Spano and C. Silva, Annu. Rev. Phys. Chem., 2014, 65, 477-500.

9 Z. El-Hachemi, C. Escudero, F. Acosta-Reyes, M. T. Casas, V. Altoe, S. Aloni, G. Oncins, A. Sorrenti, J. Crusats, J. L. Campos and J. M. Ribo, J. Mater. Chem. C, 2013, 1, 3337-3346.

10 O. Ohno, Y. Kaizu and H. Kobayashi, J. Chem. Phys., 1993, 99, 4128-4139.

11 O. Arteaga, C. Escudero, G. Oncins, Z. El-Hachemi, J. Llorens, J. Crusats, A. Canillas and J. M. Ribó, Chem Asian J., 2009, 4, 1687-1696.

12 S. C. M. Gandini, E. L. Gelamo, R. Itri and M. Tabak, Biophys. J., 2003, 85, 1259-1268.

13 S. M. Vlaming, R. Augulis, M. C. A. Stuart, J. Knoester and P. H. M. van Loosdrecht, J. Phys. Chem. B, 2009, 113, 22732283.

14 J. M. Short, J. A. Berriman, C. Kübel, Z. El-Hachemi, J.-V. Naubron and T. S. Balaban, ChemPhysChem, 2013, 14, 3209-3214. 
15 O. Arteaga, J. Freudenthal, B. Wang and B. Kahr, Appl. Opt., 2012, 51, 6805-6817.

16 O. Arteaga, M. Baldrís, J. Antó, A. Canillas, E. Pascual and E. Bertran, Appl. Opt., 2014, 53, 2236-2245.

17 O. Arteaga and A. Canillas, Opt. Lett., 2010, 35, 559561.

18 C. Escudero, Z. El-Hachemi, J. Crusats and J. M. Ribó, J. Porphyrins Phthalocyanines, 2005, 09, 852-863.

19 R. F. Pasternack and P. J. Collings, Science, 1995, 269, 935939.

20 F. Tantussi, F. Fuso, M. Allegrini, N. Micali, I. G. Occhiuto, L. M. Scolaro and S. Patane, Nanoscale, 2014, 6, 1087410878.

21 E. Malic, A. Knorr and S. Winnerl, Graphene and carbon nanotubes ultrafast relaxation dynamics and optics, Wiley, 2013.
22 Y. Wang, K. Kempa, B. Kimball, J. B. Carlson, G. Benham, W. Z. Li, T. Kempa, J. Rybczynski, A. Herczynski and Z. F. Ren, Appl. Phys. Lett., 2004, 85, 2607-2609.

23 E. Cubukcu, F. Degirmenci, C. Kocabas, M. A. Zimmler, J. A. Rogers and F. Capasso, Proc. Natl. Acad. Sci. U. S. A., 2009, 106, 2495-2499.

24 M. Y. Sfeir, F. Wang, L. Huang, C.-C. Chuang, J. Hone, S. P. O'Brien, T. F. Heinz and L. E. Brus, Science, 2004, 306, 1540-1543.

25 J. J. Crochet, J. D. Sau, J. G. Duque, S. K. Doorn and M. L. Cohen, ACS Nano, 2011, 5, 2611-2618.

26 O. Arteaga, A. Canillas, J. Crusats, Z. El-Hachemi, J. Llorens, E. Sacristan and J. M. Ribo, ChemPhysChem, 2010, 11, 3511-3516.

27 O. Arteaga, A. Canillas, R. Purrello and J. M. Ribó, Opt. Lett., 2009, 34, 2177-2179. 